\title{
Swapping science for consensus in Montreal
}

Delegates to the UN-sponsored Convention on Biological Diversity negotiated a "biosafety protocol" for the regulation of international trade in recombinant DNA-engineered plants, animals, and microorganisms for almost seven years. Before the latest meeting in Montreal last January, it appeared that many delegates would settle for any agreement, no matter how unscientific or wrong-headed, that would finally end the negotiations. And settle they did.

Instead of establishing a set of scientifically defensible, risk-based rules to govern the trans-boundary movement of what the agreement inexplicably calls "Living Modified Organisms" (a gratuitous euphemism for Genetically Manipulated Organisms, or GMOs), the parties agreed on a scheme for regulation that violates a cardinal principal of regulation - namely, that the degree of scrutiny should be commensurate with risk. Numerous international scientific organizations and policy groups have examined the known risks of biotechnology, and a widespread scientific consensus has evolved that the risk of rDNA-engineered organisms is primarily a function of the biological characteristics of individual products, not of the methods used to develop them. But biotechnology's ideological opponents have ignored these facts in crafting an agreement that singles out rDNA-engineered products solely because they have been developed with relatively new processes. The trigger for the UN's regulatory regime is merely the fact of manipulation with rDNA techniques, independent of risk. The protocol is certain to slow the pace of technological progress. It will hobble the work of academic researchers and small, innovative companies, ultimately delaying or denying the benefits of the "gene revolution" to much of the world.

The goal of the UN's biosafety protocol is ostensibly to ensure that the development, handling, transport, and field testing and use of rDNA-engineered organisms into the environment are "undertaken in a manner that prevents or reduces the risks to biological diversity, taking also into account risks to human health." It was also hoped that a multilateral agreement would promote uniformity and regulatory predictability, so that the global development of beneficial rDNA-engineered organisms would continue apace. But even a cursory examination of the protocol shows that the agreement has less to do with legitimate concerns about public health or the environment, and more to do with trade protectionism and pandering to anti-technology views.

Following the consensus reached in Montreal, the final protocol will enter into force once it is ratified by 50 member nations, which is likely to occur by the end of the summer. The question for policymakers will then become one of implementation. The agreement requires participating nations to construct regulatory systems that promote the goals of the protocol, but it leaves them much discretion in how to do so. That discretion gives government officials political cover to erect questionable barriers against rDNA-engineered organisms, but the concerted influence of the scientific community and those committed to free trade may encourage them to establish a risk-based regulatory scheme that is focused on legitimate risks.

\section{Synchronizing expectations}

Lateness is the principle cause of time-keeping grouches, but not in biotechnology. In our every day lives, we are incensed by trains delays, offended by correspondence impromptly returned, inconvenienced by deadlines missed, deflated by unmet company projections, and chided for anniversaries deprioritized. However, the complaint from the general public about biotechnology is "Too soon" rather than "Too late."

Compare the emergence of the e-world with that of modern biotechnology. The plug-and-play office-in-a-pocket did not emerge overnight. Computers rather drifted toward us. Mystical and distant tape-spinning room-sized calculators became trundling office green-screens, which in turn became graphics-led machines, which in turn became miniature devices refined to suit the needs and pocket of the home user. Even when the monsters first left their clean rooms, their introduction into the wider environment was reassuringly supervised. White-coated former operators found employment in IT support, conducting themselves in a cooperative and friendly manner to empower users and allay technophobia. Kids met computers at school, under supervision, and then sought electronic games as maintenance-free pets.

The domestication of the computer took forty years, half a human life time, two or three generations. Even now, home computer owners are still in the minority in industrialized nations. Biotechnology, in contrast, has arrived instantaneously and its penetration into the everyday world, while not as pervasive as computers, is nonetheless impressive.

Human insulin followed recombinant DNA by less than a decade and it dominated the diabetes market within a few years. Genetically manipulation in plants took longer-around a decade and a half from concept to commercial crop-but the products found their way in a matter of months into every food store. The Human Genome Project will have taken less than 15 years and most people will have noticed it only over the past five. Its impacts on healthcare, for better or worse, should be universal in the developed world within half a generation.

Biotechnologies arrive fully formed at the consumer, packaged neatly, and presented by smiling suits: sans white coat, sans help line, sans instruction manual. When they work as the consumer wants, all is well. When they don't, there is a problem.

At least some of the resentment for biotechnology from the general public has stemmed from the fact that product development times are shorter than human generational times: we can no longer rely on formal education to inform and acclimatize the populace.

There are two possible solutions to this asynchrony. One is to slow down research and development (unlikely to be a popular move in the scientific community, nor a sensible one). The other, of course, is to accelerate the introduction of the public to new biotechnologies. To accomplish this, marketing must reach back through the entire $\mathrm{R} \& \mathrm{D}$ process. And as we have insisted in this space on numerous occasions, it is imperative that scientists invent, and companies invest in, innovative ways to share knowledge with the public well before they ask them to use their products. 